\title{
ALGEBRAIC STRUCTURES OF EULER NUMBERS
}

\author{
I-CHIAU HUANG
}

(Communicated by Matthew A. Papanikolas)

Abstract. We define a finitely generated $\mathbb{Q}$-algebra $\mathfrak{E}$ with a module structure over the universal enveloping algebra of a Lie algebra. Identities of Euler numbers are investigated using the algebraic structures of $\mathfrak{E}$.

\section{INTRODUCTION}

We would like to introduce an algebraic method to study classical numbers. Such a method has been applied to Bernoulli numbers [3. In this paper, we extend the idea to Euler numbers. Let $e^{T}:=\sum_{i=0}^{\infty} T^{i} / i$ ! be an element in the power series ring $\mathbb{Q}[[T]]$ with coefficients in the field $\mathbb{Q}$ of rational numbers. Recall that the $i$-th Bernoulli number $B_{i}$ and the $i$-th Euler number $E_{i}$ are defined by power series

$$
\mathbf{B}:=\frac{T}{e^{T}-1}=\sum_{i=0}^{\infty} \frac{B_{i}}{i !} T^{i}
$$

and

$$
\mathbf{E}:=\frac{2 e^{T}}{e^{2 T}+1}=\sum_{i=0}^{\infty} \frac{E_{i}}{i !} T^{i} .
$$

We are interested in identities involving classical numbers. One of the most famous is perhaps Euler's identity for the sum of products of two Bernoulli numbers:

$$
\sum_{i=1}^{n-1}\left(\begin{array}{c}
2 n \\
2 i
\end{array}\right) B_{2 i} B_{2 n-2 i}=-(2 n+1) B_{2 n},
$$

where $n \geq 2$. Euler's identity has been generalized to sums of products of more Bernoulli numbers 1, 6, 8, 2, For instance,

$$
\sum_{i+j+k=n}\left(\begin{array}{c}
2 n \\
2 i, 2 j, 2 k
\end{array}\right) B_{2 i} B_{2 j} B_{2 k}=(n+1)(2 n+1) B_{2 n}+\frac{1}{2} n(2 n-1) B_{2 n-2},
$$

where $n \geq 3$. An interpretation of these formulae in terms of complete sums is given in 4. Euler's identity has other variations such as

$$
\sum_{i=0}^{n}\left(\begin{array}{l}
n \\
i
\end{array}\right) B_{1+i} B_{1+n-i}=\frac{1}{6}(n-1) B_{n}-B_{n+1}-\frac{1}{6}(n+3) B_{n+2} .
$$

See [1] for more similar identities. The new viewpoint of [3] on the above identities is that they all come from algebraic relations of elements in a commutative ring

Received by the editors November 9, 2010.

2010 Mathematics Subject Classification. Primary 11B68; Secondary 16S30.

Key words and phrases. Euler number, identity, Lie algebra, skew polynomial ring, universal enveloping algebra. 
with a module structure over the Weyl algebra $\mathbb{Q}\langle T, d / d T\rangle$. We remark that the Weyl algebra is a skew polynomial ring. For the definitions and basic properties of Weyl algebras and skew polynomial rings, the reader is referred to [5, Chapter 1].

In this paper, we define a subring $\mathfrak{E}$ of $\mathbb{Q}[[T]]$ containing $\mathbf{E}$ with a module structure over another skew polynomial ring, which is the universal enveloping algebra of a two-dimensional Lie algebra. See [5, Chapter 1] for definitions and constructions of universal enveloping algebras and Lie algebras. Via algebraic relations of elements in $\mathfrak{E}$, we reproduce formulae in 9 for the sum of products of $2 m+1$ Euler numbers such as

$$
\sum_{i+j+k=n}\left(\begin{array}{c}
2 n \\
2 i, 2 j, 2 k
\end{array}\right) E_{2 i} E_{2 j} E_{2 k}=\frac{1}{2} E_{2 n}-\frac{1}{2} E_{2 n+2} .
$$

See 9 also for explicit formulae of the sum of products of five, seven and nine Euler numbers. Analyzing the algebraic structures of $\mathfrak{E}$, we show that such a type of formula does not exist for the sum of products of $2 m$ Euler numbers. Our method gives numerous identities of Euler numbers; some are elegant and some can be complicated. Among them, we have

$$
\sum_{i+j+k=n}\left(\begin{array}{c}
n \\
i, j, k
\end{array}\right) E_{i} E_{1+j} E_{1+k}=\frac{1}{8} E_{n}-\frac{1}{12} E_{n+2}-\frac{1}{24} E_{n+4}
$$

and

$$
\sum_{i+j+k=n}\left(\begin{array}{c}
n \\
i, j, k
\end{array}\right) E_{2+i} E_{j} E_{k}=-\frac{1}{4} E_{n}+\frac{1}{3} E_{n+2}-\frac{1}{12} E_{n+4} .
$$

As in 3] for Bernoulli polynomials, one may extend the algebraic structures considered in this paper to include Euler polynomials. It is also plausible that there exist algebraic structures accommodating two or more classical numbers (for instance Bernoulli numbers and Euler numbers) simultaneously. We leave the possibility to the reader.

\section{Algebraic structures}

Definition 2.1. We define $\mathfrak{E}$ to be the $\mathbb{Q}$-subalgebra of $\mathbb{Q}[[T]]$ generated by $x:=e^{T}$ and $y:=\mathbf{E}$.

To understand the ring structure of $\mathfrak{E}$, we consider the $\mathbb{Q}$-algebra homomorphism

$$
\pi: \mathbb{Q}[X, Y] \rightarrow \mathbb{Q}[[T]]
$$

given by $\pi(X)=e^{T}$ and $\pi(Y)=\mathbf{E}$. Clearly, $\pi\left(X^{2} Y+Y-2 X\right)=0$.

Proposition 2.2. The $\mathbb{Q}$-algebra $\mathfrak{E}$ is isomorphic to $\mathbb{Q}[X, Y] /\left(X^{2} Y+Y-2 X\right)$.

Proof. It suffices to prove that the kernel of $\pi$ is generated by $X^{2} Y+Y-2 X$. The image of $\pi$ is contained in the quotient field of $\mathbb{Q}\left[e^{T}\right]$. Since $e^{T}$ is transcendental over $\mathbb{Q}$, to prove the proposition, it suffices to prove that the kernel of the $\mathbb{Q}[X]$-algebra homomorphism

$$
\tilde{\pi}: \mathbb{Q}[X, Y] \rightarrow \mathbb{Q}(X)
$$

sending $Y$ to $2 X /\left(X^{2}+1\right)$ is generated by $X^{2} Y+Y-2 X$ as well. Consider an element in the kernel of $\tilde{\pi}$. Replacing $X^{2} Y$ by $2 X-Y$ repeatedly, we can write the element in the form $f(X) X+g(Y) Y X+h(Y)$ for some one-variable polynomials 
$f, g$ and $h$ over $\mathbb{Q}$. It suffices to show that $f(X) X+g(Y) Y X+h(Y)=0$. We work on the field $\mathbb{Q}\left(\left(X^{-1}\right)\right)$ of Laurent series in the variable $X^{-1}$ : In

$$
f(X) X+g\left(\frac{2 X^{-1}}{1+X^{-2}}\right) \frac{2}{1+X^{-2}}+h\left(\frac{2 X^{-1}}{1+X^{-2}}\right)=0
$$

only $f(X) X$ consists of positive powers of $X$. Hence $f(X)=0$. Now we work on the field $\mathbb{Q}((X))$ of Laurent series in the variable $X$ : The constant term on the left-hand side of

$$
g\left(\frac{2 X}{X^{2}+1}\right) \frac{2 X^{2}}{X^{2}+1}+h\left(\frac{2 X}{X^{2}+1}\right)=0
$$

is the constant term of $h(Y)$. Hence $h(0)=0$. Back to the field $\mathbb{Q}\left(\left(X^{-1}\right)\right)$ : The constant term on the left-hand side of

$$
g\left(\frac{2 X^{-1}}{1+X^{-2}}\right) \frac{2}{1+X^{-2}}+h\left(\frac{2 X^{-1}}{1+X^{-2}}\right)=0
$$

is twice the constant term of $g(Y)$. Hence $g(0)=0$. So we can factor out $Y$ from $g(Y)$ and $h(Y)$. Induction on the degree of $h(Y)$ shows that $h(Y)=0$. Now $g(Y) Y X \in \operatorname{ker} \tilde{\pi}$; hence $g\left(2 X /\left(X^{2}+1\right)\right)=0$. This implies that $g(Y)=0$, since $2 X /\left(X^{2}+1\right)$ is transcendental over $\mathbb{Q}$.

Definition 2.3. Let $U$ be the ring of endomorphisms of $\mathbb{Q}[[T]]$ generated by the multiplication map $x:=e^{T}$ and the derivation $\delta:=d / d T$. We denote the action of $\varphi \in U$ on $f \in \mathbb{Q}[[T]]$ by $\varphi \cdot f$.

Here we use the notation $x$ both for an element in $\mathfrak{E}$ and an element in $U$ without confusion. Hence a polynomial in $x$ may stand for an element in $\mathfrak{E}$ or an element in $U$ depending on the context. The relations

$$
\begin{aligned}
& \delta \cdot x=x, \\
& \delta \cdot y=y-x y^{2}
\end{aligned}
$$

in $\mathfrak{E}$ imply that $\mathfrak{E}$ is stable under the action of $U$. In other words, $\mathfrak{E}$ is a $U$-module. As a special case of the Leibniz rule, we have the relation

$$
\delta x-x \delta=x
$$

in $U$. In general, one can show that

$$
\begin{aligned}
\delta^{n} x^{m} & =x^{m}(m+\delta)^{n}, \\
x^{m} \delta^{n} & =(\delta-m)^{n} x^{m}
\end{aligned}
$$

by induction. Consider $f_{n}, f_{n+1}, \ldots, f_{n+m} \in \mathbb{Q}[x]$. If $f_{n}$ does not vanish, then neither does the linear combination $\varphi=f_{n} \delta^{n}+f_{n+1} \delta^{n+1}+\cdots+f_{n+m} \delta^{n+m}$, since $n ! f_{n}=\varphi \cdot T^{n}$. Thus every element in $U$ can be written uniquely as a $\mathbb{Q}$-linear combination of elements of the form $x^{m} \delta^{n}$. Using relation (2.1), one shows also that every element in $U$ can be written uniquely as a $\mathbb{Q}$-linear combination of elements of the form $\delta^{n} x^{m}$. Using relation (2.2) for $n=1$, one sees that $U$ is isomorphic to the skew polynomial ring $\mathbb{Q}[x][\delta ;-x(d / d x)]$. Note that $U$ is also isomorphic to the universal enveloping algebra of the two-dimensional Lie algebra $\mathfrak{g}$ with basis $\{\delta, x\}$ and Lie bracket $[\delta, x]=x$. See $[5,1.7 .6]$.

The ideal $\mathfrak{E} y$ of $\mathfrak{E}$ is also a $U$-submodule of $\mathfrak{E}$. To understand algebraic meanings of identities of Euler numbers, we need to find generators and relations of $\mathfrak{E} y$ as a $U$-module. We begin with a comparison between the $\mathfrak{E}$-ideal and the $U$-submodule generated by a power of $y$. 
Proposition 2.4. $\mathfrak{E} y^{m}=U \cdot y^{m}$ for $m \geq 1$.

Proof. Clearly, $x^{n} \cdot y^{m}=x^{n} y^{m}$ for any $n$ and $\delta \cdot y^{m}=m(1-x y) y^{m}$. Induction on $n$ shows that $\delta^{n} \cdot y^{m} \in \mathfrak{E} y^{m}$. Thus $U \cdot y^{m} \subset \mathfrak{E} y^{m}$. To prove $\mathfrak{E} y^{m} \subset U \cdot y^{m}$, we consider the derivation $x \delta$ on $\mathfrak{E}$. Note that

$$
(x \delta) \cdot y^{m}=m y^{m-1} x\left(y-x y^{2}\right)=m x y^{m}-m(2 x-y) y^{m}=m y^{m+1}-m x y^{m},
$$

which can also be written as

$$
m ! y^{m+1}=(m-1) ! x(\delta+m) \cdot y^{m} .
$$

Indeed the above relation is a special case of

$$
(n !) y^{n+1}=(m-1) ! x^{n-m+1}(\delta+m)(\delta+m+2) \cdots(\delta+2 n-m) \cdot y^{m}
$$

for $n \geq m \geq 1$, which implies that $\mathfrak{E} y^{m} \subset U \cdot y^{m}$. Relation (2.3) can be proved by induction on $n$ : Assume that it holds for an $n$. Applying the derivation $x \delta$ on (2.3), we have

$$
(n+1) ! y^{n}\left(y^{2}-x y\right)=(m-1) ! x \delta x^{n-m+1}(\delta+m)(\delta+m+2) \cdots(\delta+2 n-m) \cdot y^{m} .
$$

Using the relation $\delta x^{n-m+1}=x^{n-m+1}(\delta+n-m+1)$, we have

$$
\begin{aligned}
(n+1) & ! y^{n+2} \\
= & (m-1) ! x^{n-m+2}(n+1)(\delta+m)(\delta+m+2) \cdots(\delta+2 n-m) \cdot y^{m} \\
& +(m-1) ! x^{n-m+2}(\delta+n-m+1)(\delta+m)(\delta+m+2) \cdots(\delta+2 n-m) \cdot y^{m} \\
= & (m-1) ! x^{n-m+2}(\delta+m)(\delta+m+2) \cdots(\delta+2 n+2-m) \cdot y^{m} .
\end{aligned}
$$

The $\mathfrak{E}$-ideal $\operatorname{Ann}_{\mathfrak{E}}(y):=\{f \in \mathfrak{E}: y f=0\}$ consists of only 0. However the left $U$-ideal

$$
\operatorname{Ann}_{U}(y):=\{f \in U: f \cdot y=0\}
$$

consists of non-trivial elements. To compute $\operatorname{Ann}_{U}(y)$, we need to study the set $\left\{\delta^{i} \cdot y^{m}\right\}_{i \geq 0}$ for $m=1$ and 2 .

Lemma 2.5. Given $m \geq 1$, the elements of the set $\left\{\delta^{i} \cdot y^{m}\right\}_{i \geq 0}$ are linearly independent over $\mathbb{Q}$.

Proof. Consider a $\mathbb{Q}$-linear relation

$$
a_{0} y^{m}+a_{1} \delta \cdot y^{m}+a_{2} \delta^{2} \cdot y^{m}+\cdots+a_{n} \delta^{n} \cdot y^{m}=0 .
$$

Since $\delta^{k}$ can be written uniquely as a $\mathbb{Q}$-linear combination of 1 and the elements of the form $\prod_{i=1}^{j}(\delta+m+2 i-2)$ with $1 \leq j \leq k$, we may rewrite (2.4) as

$$
a_{0}^{\prime} y^{m}+\sum_{j=1}^{n} a_{j}^{\prime} \prod_{i=1}^{j}(\delta+m+2 i-2) \cdot y^{m}=0
$$

for some $a_{j}^{\prime} \in \mathbb{Q}$. If relation (2.4) is not trivial, say $a_{n} \neq 0$, then neither is (2.5) since $a_{n}^{\prime}=a_{n}$. Multiplying (2.5) by $x^{n}$ and using (2.3), we obtain

$$
a_{0}^{\prime} x^{n} y^{m}+\sum_{j=1}^{n} a_{j}^{\prime} \frac{(m+j-1) !}{(n-j) !} x^{n-j} y^{m+j}=0 .
$$


If (2.4) is not trivial, the relation

$$
\frac{a_{0}^{\prime} 2^{m}}{\left(1+X^{2}\right)^{m}}+\sum_{j=1}^{n} \frac{a_{j}^{\prime}(m+j-1) ! 2^{m+j}}{(n-j) !\left(1+X^{2}\right)^{m+j}}=0
$$

contradicts the fact that $1 /\left(1+X^{2}\right)$ is transcendental over $\mathbb{Q}$.

Lemma 2.6. $\delta^{n} \cdot y^{m} \in \mathbb{Z}[x y] y^{m}$ for $m, n \geq 1$.

Proof. Let $\phi_{m 1}:=m(1-X)$ and

$$
\phi_{m(n+1)}:=m(1-X) \phi_{m n}+\left(2 X-X^{2}\right) \frac{d \phi_{m n}}{d X}
$$

for $n \geq 1$. Induction on $n$ gives $\delta^{n} \cdot y^{m}=y^{m} \phi_{m n}(x y)$.

Proposition 2.7. $\operatorname{Ann}_{U}(y)=U(\delta-1)\left(x^{2}+1\right)$.

Proof. Clearly,

$$
(\delta-1)\left(x^{2}+1\right) \cdot y=(\delta-1) \cdot 2 x=0 .
$$

Hence $U(\delta-1)\left(x^{2}+1\right) \subset \operatorname{Ann}_{U}(y)$. Recall that an element in $U$ can be written as $\sum a_{i j} \delta^{i} x^{j}$ for $a_{i j} \in \mathbb{Q}$. Replacing $\delta x^{2}$ by $x^{2}-\delta+1$ repeatedly, to prove $\operatorname{Ann}_{U}(y) \subset$ $U(\delta-1)\left(x^{2}+1\right)$, it suffices to consider an element $f(x) x+g(\delta) \delta x+h(\delta) \in \operatorname{Ann}_{U}(y)$ for some one-variable polynomials $f, g$ and $h$ over $\mathbb{Q}$. We need to prove that $f(x) x+g(\delta) \delta x+h(\delta)=0$. By abusing the notation, we denote by

$$
\tilde{\pi}: \mathfrak{E} \rightarrow \mathbb{Q}(X)
$$

also the $\mathbb{Q}$-algebra homomorphism sending $x$ and $y$ to $X$ and $2 X /\left(X^{2}+1\right)$, respectively. The action of $f(x) x+g(\delta) \delta x+h(\delta)$ on $y$ gives

$$
f(X) \frac{2 X^{2}}{X^{2}+1}+\tilde{\pi}(g(\delta) \delta x \cdot y)+\tilde{\pi}(h(\delta) \cdot y)=0 .
$$

Note that

$$
\delta x \cdot y=x\left(y-x y^{2}\right)+y x=2 x y-(2 x-y) y=y^{2} .
$$

So we can write

$$
2 f(X)+\left(1+X^{-2}\right) \tilde{\pi}\left(g(\delta) \cdot y^{2}\right)+\left(1+X^{-2}\right) \tilde{\pi}(h(\delta) \cdot y)=0
$$

as elements in $\mathbb{Q}\left(\left(X^{-1}\right)\right)$. Note also that

$$
\begin{aligned}
\tilde{\pi}\left(y^{2}\right) & =4 X^{-2} /\left(1+X^{-2}\right)^{2}, \\
\tilde{\pi}(x y) & =2 /\left(1+X^{-2}\right), \\
\tilde{\pi}(y) & =2 X^{-1} /\left(1+X^{-2}\right) .
\end{aligned}
$$

By Lemma 2.6, the power series $\left(1+X^{-2}\right) \tilde{\pi}\left(g(\delta) \cdot y^{2}\right)$ has non-trivial coefficients only at even and non-zero powers of $X^{-1}$, and the power series $\left(1+X^{-2}\right) \tilde{\pi}(h(\delta) \cdot y)$ has non-trivial coefficients only at odd powers of $X^{-1}$. Therefore

$$
f(X)=\tilde{\pi}\left(g(\delta) \cdot y^{2}\right)=\tilde{\pi}(h(\delta) \cdot y)=0,
$$

as well as

$$
f(x)=g(\delta) \cdot y^{2}=h(\delta) \cdot y=0 .
$$

By Lemma 2.5, $g(\delta)=h(\delta)=0$. 
Combining Proposition 2.4 and Proposition 2.7 the $U$-module structure of $\mathfrak{E} y$ is well-understood:

Theorem 2.8. $\mathfrak{E} y \simeq U / U(\delta-1)\left(x^{2}+1\right)$.

\section{IDENTITIES}

Equating coefficients of $T^{n}$ from a relation between $x$ and $y$, we obtain an identity of Euler numbers. By Proposition 2.2, there is essentially only one identity of Euler numbers coming exclusively from the ring structure of $\mathfrak{E}$ :

\section{Identity 3.1.}

$$
\sum_{i=0}^{n}\left(\begin{array}{c}
n \\
i
\end{array}\right) 2^{n-i} E_{i}=2-E_{n}
$$

With the $U$-module structure, there are other interesting algebraic relations.

Proposition 3.2. $y^{2 n+1} \in \mathbb{Q}[\delta] \cdot y$ for $n \geq 1$.

Proof. We begin with

$$
\delta^{2} \cdot y^{n}=\delta \cdot\left(n y^{n}-n x y^{n+1}\right)=n^{2} y^{n}-n(n+1) y^{n+2},
$$

which can also be written as

$$
n(n+1) y^{n+2}=\left(n^{2}-\delta^{2}\right) \cdot y^{n} .
$$

Using relation (3.1) repeatedly for odd powers of $y$, we obtain

$$
y^{2 n+1}=\frac{\left(1-\delta^{2}\right)\left(3^{2}-\delta^{2}\right) \cdots\left((2 n-1)^{2}-\delta^{2}\right)}{(2 n) !} \cdot y .
$$

We recover the identities obtained in 9] from relation (3.2). Below are formulae for the sum of products of three and five Euler numbers.

Identity 3.3.

$$
\sum_{i+j+k=n}\left(\begin{array}{c}
n \\
i, j, k
\end{array}\right) E_{i} E_{j} E_{k}=\frac{1}{2} E_{n}-\frac{1}{2} E_{n+2} .
$$

Identity 3.4.

$$
\sum_{i+j+k+l+m=n}\left(\begin{array}{c}
n \\
i, j, k, l, m
\end{array}\right) E_{i} E_{j} E_{k} E_{l} E_{m}=\frac{3}{8} E_{n}-\frac{5}{12} E_{n+2}+\frac{1}{24} E_{n+4} .
$$

The following proposition explains why there is no analog to Identity 3.3 and Identity 3.4 for the sum of products of $2 n$ Euler numbers.

Proposition 3.5. $y^{2 n} \notin \mathbb{Q}[\delta] \cdot y$ for $n \geq 1$.

Proof. We use relation (2.3) with $m=1$ :

$$
(2 n-1) ! y^{2 n}=x^{2 n-1}(\delta+1)(\delta+3) \cdots(\delta+4 n-3) \cdot y .
$$

If $y^{2 n} \in \mathbb{Q}[\delta] \cdot y$, combining (3.3) and Lemma 2.6 with $m=1$, there would exist $\eta \in \mathbb{Q}(X)$ such that $x^{2 n-1}=\eta(x y)$. This is impossible, since the right-hand side of the equation

$$
X^{2 n-1}=\eta\left(\frac{2 X^{2}}{X^{2}+1}\right)
$$

consists of only even powers of $X$. 
Actions by $x$ and $\delta$ together give formulae for the sum of products of $2 n$ Euler numbers.

Proposition 3.6. $y^{2 n} \in \mathbb{Q}[\delta] x \cdot y$ for $n \geq 1$.

Proof. The proposition for the case $n=1$ is shown in (2.6). For $n>1$, applying relation (3.1) repeatedly for even powers of $y$, we obtain

$$
y^{2 n}=\frac{\left(2^{2}-\delta^{2}\right)\left(4^{2}-\delta^{2}\right) \cdots\left((2 n-2)^{2}-\delta^{2}\right) \delta x}{(2 n-1) !} \cdot y .
$$

Below are identities obtained from relation (2.6) and relation (3.4) with $n=2$.

\section{Identity 3.7.}

$$
\sum_{i=0}^{n}\left(\begin{array}{c}
n \\
i
\end{array}\right) E_{i} E_{n-i}=\sum_{i=0}^{n+1}\left(\begin{array}{c}
n+1 \\
i
\end{array}\right) E_{i}
$$

\section{Identity 3.8.}

$$
\sum_{i+j+k+l=n}\left(\begin{array}{c}
n \\
i, j, k, l
\end{array}\right) E_{i} E_{j} E_{k} E_{l}=\frac{2}{3} \sum_{i=0}^{n+1}\left(\begin{array}{c}
n+1 \\
i
\end{array}\right) E_{i}-\frac{1}{6} \sum_{i=0}^{n+3}\left(\begin{array}{c}
n+3 \\
i
\end{array}\right) E_{i} .
$$

Elegant identities are obtained from polynomial relations of elements in $\mathbb{Q}[\delta] \cdot y$. Recall Proposition 3.2 that $Q[\delta] \cdot y$ contains all odd powers of $y$. So the following proposition gives rise to a method to produce interesting identities.

Proposition 3.9. If the sequence $i_{1}, \ldots, i_{n}$ consists of an even number of odd integers and an odd number of even integers, then $\left(\delta^{i_{1}} \cdot y\right)\left(\delta^{i_{2}} \cdot y\right) \cdots\left(\delta^{i_{n}} \cdot y\right) \in \mathbb{Q}\left[y^{2}\right] y$.

Proof. It suffices to prove $\left(\delta^{2 n-1} \cdot y\right)\left(\delta^{2 m-1} \cdot y\right) \in \mathbb{Q}\left[y^{2}\right]$ and $\delta^{2 n} \cdot y \in \mathbb{Q}\left[y^{2}\right] y$ for $m, n \geq 1$. Let $f_{1}:=1-2 T$ and, for $n>0$, let

$$
f_{n+1}:=(1-2 T) f_{n}+\left(8 T-10 T^{2}\right) f_{n}^{\prime}+\left(4 T^{2}-4 T^{3}\right) f_{n}^{\prime \prime} .
$$

Induction on $n$ shows that $\delta^{2 n} \cdot y=y f_{n}\left(y^{2}\right)$. Let $g_{1}:=1$ and, for $n>1$, let

$$
g_{n}:=f_{n-1}+2 T f_{n-1}^{\prime} .
$$

It is straightforward to compute that $\left(\delta^{2 n-1} \cdot y\right)\left(\delta^{2 m-1} \cdot y\right)=\left(y^{2}-y^{4}\right) g_{n}\left(y^{2}\right) g_{m}\left(y^{2}\right)$.

Here are two examples from the relations

$$
(\delta \cdot y)^{2} y=y^{3}-y^{5}=\left(\frac{1}{8}-\frac{1}{12} \delta^{2}-\frac{1}{24} \delta^{4}\right) \cdot y
$$

and

$$
\left(\delta^{2} \cdot y\right) y^{2}=y^{3}-2 y^{5}=\left(-\frac{1}{4}+\frac{1}{3} \delta^{2}-\frac{1}{12} \delta^{4}\right) \cdot y .
$$

Identity 3.10 .

$$
\sum_{i+j+k=n}\left(\begin{array}{c}
n \\
i, j, k
\end{array}\right) E_{1+i} E_{1+j} E_{k}=\frac{1}{8} E_{n}-\frac{1}{12} E_{n+2}-\frac{1}{24} E_{n+4} .
$$


Identity 3.11 .

$$
\sum_{i+j+k=n}\left(\begin{array}{c}
n \\
i, j, k
\end{array}\right) E_{2+i} E_{j} E_{k}=-\frac{1}{4} E_{n}+\frac{1}{3} E_{n+2}-\frac{1}{12} E_{n+4} .
$$

Identities of Euler numbers can be obtained from a known identity through an element in $\operatorname{Ann}_{U}(y)$. For instance, the element

$$
x^{2}\left(\delta^{2}+4 \delta+3\right)-\left(1-\delta^{2}\right)=\left(\delta^{2}-1\right)\left(x^{2}+1\right) \in \operatorname{Ann}_{U}(y)
$$

gives a new guise of Identity 3.3 .

\section{Identity 3.12.}

$$
\sum_{i+j+k=n}\left(\begin{array}{c}
n \\
i, j, k
\end{array}\right) E_{i} E_{j} E_{k}=\sum_{i=0}^{n} 2^{n-i}\left(\begin{array}{c}
n \\
i
\end{array}\right)\left(\frac{1}{2} E_{i+2}+2 E_{i+1}+\frac{3}{2} E_{i}\right) .
$$

Note that all odd Euler numbers are zero, since $2 e^{-T} /\left(e^{-2 T}+1\right)=\mathbf{E}$. So all identities in this section can be rewritten in terms of Euler numbers with even indices. In particular, Identity 3.7 and Identity 3.8 with $n=2 m-1$ give rise to the following recurrence formulae for Euler numbers.

Identity 3.13.

$$
\sum_{i=0}^{m}\left(\begin{array}{c}
2 m \\
2 i
\end{array}\right) E_{2 i}=0
$$

\section{Identity 3.14 .}

$$
E_{2 m+2}=\sum_{i=0}^{m} \frac{6 m^{2}-16 m i+8 i^{2}+9 m-12 i+3}{(m+1)(2 m+1)}\left(\begin{array}{c}
2 m+2 \\
2 i
\end{array}\right) E_{2 i} .
$$

\section{REFERENCES}

[1] T. Agoh and K. Dilcher. Convolution identities and lacunary recurrences for Bernoulli numbers. J. Number Theory, 124(1):105-122, 2007. MR2320993 (2008d:11019)

[2] K. Dilcher. Sums of products of Bernoulli numbers. J. Number Theory, 60(1):23-41, 1996. MR:1405723 (97h:11014)

[3] I-C. Huang. Algebraic structures of Bernoulli numbers and polynomials. arXiv:1005.0177.

[4] I-C. Huang and S.-Y. Huang. Bernoulli numbers and polynomials via residues. J. Number Theory, 76(2):178-193, 1999. MR.1684682(2000d:11027)

[5] J. C. McConnell and J. C. Robson. Noncommutative Noetherian rings, volume 30 of Graduate Studies in Mathematics. American Mathematical Society, Providence, RI, revised edition, 2001. MR1811901 (2001i:16039)

[6] A. Sankaranarayanan. An identity involving Riemann zeta function. Indian J. Pure Appl. Math., 18(7):794-800, 1987. MR908205 (88i:11059)

[7] R. Sitaramachandrarao and B. Davis. Some identities involving the Riemann zeta function. II. Indian J. Pure Appl. Math., 17(10):1175-1186, 1986. MR864156 (88d:11080)

[8] W.-P. Zhang. On the several identities of Riemann zeta-function. Chinese Sci. Bull., 36(22):1852-1856, 1991. MR:1150577 (92m:11086)

[9] W.-P. Zhang. Some identities for Euler numbers. J. Northwest Univ., 22(1):17-20, 1992. MR 1166105 (93h:11024)

Institute of Mathematics, Academia Sinica, 6F, Astronomy-Mathematics Building, No. 1, Sec. 4, Roosevelt Road, Taipei 10617, Taiwan, Republic of China

E-mail address: ichuang@math.sinica.edu.tw 